\title{
Recent advances in sample preparation techniques to overcome difficulties encountered during quantitative analysis of small molecules from biofluids using LC-MS/MS
}

Cite this: Analyst, 2014, 139, 2265

\begin{abstract}
Caroline Bylda, ${ }^{a b}$ Roland Thiele, ${ }^{a}$ Uwe Kobold $^{a}$ and Dietrich A. Volmer ${ }^{* b}$
Liquid chromatography-mass spectrometry analysis of small molecules from biofluids requires sensitive and robust assays. Because of the very complex nature of many biological samples, efficient sample preparation protocols to remove unwanted components and to selectively extract the compounds of interest are an essential part of almost every bioanalytical workflow. This review describes the most common problems encountered during sample preparation, ways to optimize established sample preparation techniques and important recent developments to reduce or eliminate major interferents from biofluids.
\end{abstract}

Received 14th January 2014

Accepted 12th February 2014

DOI: $10.1039 / c 4 a n 00094 c$

www.rsc.org/analyst

levels, co-components of the sample matrix can influence the

\section{Introduction}

The primary goal of sample preparation is to isolate one or several target analytes from the other components of the sample mixture (matrix). Depending on their nature and concentration

${ }^{a}$ Roche Diagnostics GmbH, Penzberg, Germany

${ }^{b}$ Institute of Bioanalytical Chemistry, Saarland University, D-66123 Saarbrücken, Germany. E-mail: Dietrich.Volmer@mx.uni-saarland.de; Fax: +49 681302 2963; Tel: +496813023433

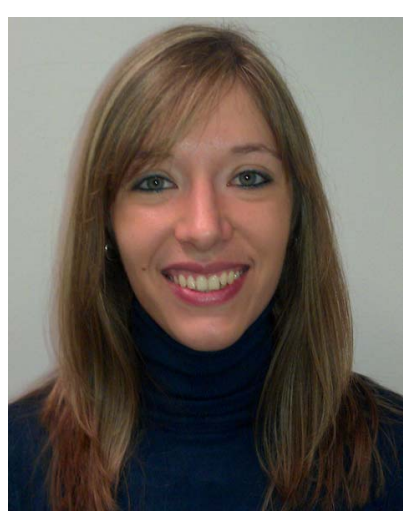

Caroline Bylda is a PhD student in Professor Volmer's group at the Institute of Bioanalytical Chemistry, Saarland University, in Saarbrücken, Germany. In 2011, she obtained her Master's degree after completing a research thesis on the $L C-M S$ / MS analysis of $\Delta^{9}$-tetrahydrocannabinol and its metabolites in oral fluid. Her current PhD project is a collaborative effort with Roche Diagnostics in Penzberg, Germany, and focusses on LC-MS/MS methods for quantitative analysis of small molecules in biofluids. quantitation of target analyte(s) during subsequent liquid chromatography-mass spectrometry (LC-MS) or tandem mass spectrometry (LC-MS/MS) experiments if not removed prior to analysis. The development of new LC-MS/MS methods for small molecules in biological fluids is becoming increasingly more challenging, because of the need to continuously achieve higher sensitivity and better assay robustness in complex biofluids such as serum, plasma, urine, oral fluid or cerebrospinal fluid (CSF). In addition, because of the very low concentration levels

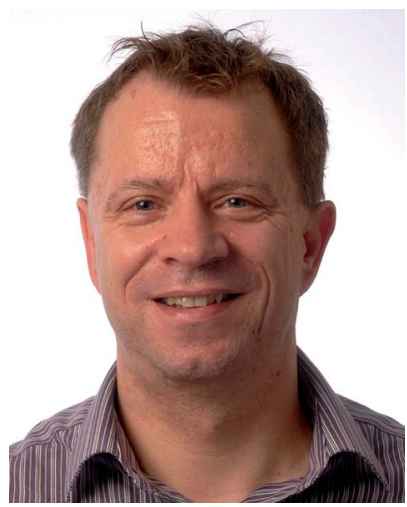

Dietrich A. Volmer is Krupp Professor and Chair in Analytical Chemistry, and Director of the Institute of Bioanalytical Chemistry in the Department of Chemistry at Saarland University in Saarbrücken, Germany. He received his $P h D$ in Analytical Chemistry from the University of Hannover, Germany. After postdoctoral research in Jefferson (AR, USA), he held academic appointments in Halifax (NS, Canada) and Cambridge (UK), before moving to Saarbrücken. Dietrich Volmer has also been Editor of Rapid Communications in Mass Spectrometry since 2004. His current research program focuses on bioanalytical chemistry, in particular biological mass spectrometry, quantification techniques, gas phase ion chemistry, chemical separations, imaging mass spectrometry, biomarkers and metabolomics. 
of pharmaceutical targets, samples often need to be preconcentrated before analysis. Unfortunately, this does not only increase the concentration of the desired compound in the sample extract but also often raises the levels of interfering components. As a result, very specific and effective sample clean-up procedures are required for sensitive and selective LCMS/MS assays today. ${ }^{1}$

This short review presents the main difficulties encountered during sample preparation for analysis of small molecules from biofluids by LC-MS/MS and summarizes several critical factors that particular attention should be paid to, followed by an overview of the latest developments in sample preparation techniques to overcome common difficulties with complex biofluids.

\section{Matrix effects}

The general term used today to describe problems encountered during analysis of complex biological samples is "matrix effects". These effects are usually caused by endogenous (e.g. metabolites of the target analyte, proteins or lipids) or exogenous (all substances introduced during sample processing and analysis) compounds. Depending on their chemical properties, it may or may not be necessary to remove all of these interferents from the sample before injection into the LC-MS system. Also, only matrix compounds coeluting with target analytes during the chromatographic separation prior to MS analysis can cause a change in the response of the analyte, either positive (ion enhancement effect) or negative (ion suppression effect). ${ }^{2}$

Different methods have been presented to examine matrix effects. A common approach is the post-extraction spike method, ${ }^{3-5}$ where the peak area of the target analyte that has been spiked into the biological matrix prior to the sample preparation is compared to the area of the same analyte spiked post-extraction into the biological fluid extract. The ratio between the two values represents the absolute matrix effect. The relative matrix effect is determined by comparing several lots of the biological matrix. ${ }^{3}$ Obviously, both absolute and relative matrix effects depend strongly on the target analyte and the ionization technique used for LC-MS/MS.

Another popular method is post-column infusion, ${ }^{6-8}$ where possible matrix effects are assessed by continuous post-column infusion of the analyte after injection of a processed blank serum sample onto the chromatography column. Any variation of signal intensity at or near the retention times of the analyte would indicate the presence of substances from the matrix interfering with the analysis.

Matrix effects have been shown to be dependent on the ionization methods used for the LC-MS method, ${ }^{3}$ which are usually either electrospray ionization (ESI) or atmospheric pressure chemical ionization (APCI) in most modern LC-MS/MS assays. The chemical structures and the concentration levels of both analyte and co-eluting mixture components determine whether they outcompete each other during the ionization process. ${ }^{9}$ For example, ESI is particularly sensitive to co-eluting phospholipids because ESI is strongly biased towards surfactants, ${ }^{10}$ which enrich at the surface of the droplets during the liquid/gas-phase ion transfer. That is, phospholipids at the surface of droplets can inhibit ejection of analyte ions trapped inside the droplets. On the other hand, APCI is often less affected by suppression effects, as there is no competition between compounds to enter the gas-phase of the mass spectrometer. Nevertheless, APCI still experiences matrix effects in multicomponent samples. As biofluids contain numerous endogenous molecules, often at high levels, with potentially very high basicities and surface activities, ion suppression effects will almost always be present in any LC-MS/MS assay.

Different strategies are available to eliminate or reduce matrix effects. One approach is to optimize the chromatographic separation to separate the analytes from interfering compounds. ${ }^{1,11,12}$ This can, however, result in long chromatographic run times. Another approach is to optimize the sample preparation, to obtain clean extracts of the target analytes. With proper sample preparation and the use of isotopically labeled standards, many matrix effects can be eliminated or strongly reduced. Some cases remain, however, where the high variability of the matrix composition makes the use of standard addition calibration necessary. ${ }^{13-16}$

There are several well-known causes for matrix effects in the analysis of clinically-relevant substances from biological samples. For example, hemolyzed or lipaemic samples have great influence on the analysis of serum and plasma samples. ${ }^{17,18}$ Cases also have been reported, where buffers used for solid-phase extraction (SPE) triggered matrix effects in LC-MS/MS. ${ }^{19}$ The most important interferents, however, are phospholipids, which not only affect MS response of many analytes greatly, but which are also very difficult to remove from the samples.

\section{Phospholipids}

Phospholipids (PPL) are major constituents of cell membranes and are therefore very abundant in serum and plasma. ${ }^{20}$ They consist of two functional groups: a hydrophilic head group composed of phosphate and choline units, and a hydrophobic tail, made up of fatty acyl chains. The most abundant phospholipids are glycerophosphocholines (GPChos) ( $70 \%$ of total phospholipids) and lysophosphatidylcholines (10\% of total phospholipids) (Fig. 1). ${ }^{11}$ These two groups are known to cause serious ion suppression effects in LC-MS analysis, caused by competition for space on the surface of droplets formed during

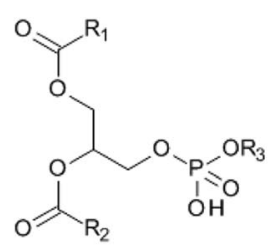

Glycerophospholipids

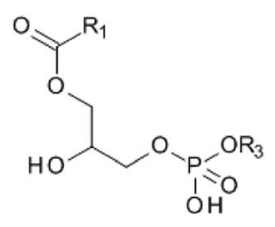

Lysophospholipids
Fig. 1 Chemical structures of the two most important groups of phospholipids. 
the ESI process (vide supra). ${ }^{3,10}$ Phospholipids are present at different concentration levels in serum and plasma samples, depending on the sampling device used..$^{21} \mathrm{~A}$ very simple method to monitor possible ion suppression effects from GPCho was described by Little et al. as in-source multiple reaction monitoring (IS-MRM). ${ }^{22}$ Using the positive ion mode, a common product ion for the most abundant GPCho is trimethylammonium-ethyl phosphate at $\mathrm{m} / \mathrm{z} 184$, which was monitored during analysis of an analyte-free sample. This class-specific product ion was generated using in-source dissociation of the eluting GPCho during the chromatographic run. ${ }^{22}$ Other methods have been described that allow screening for less abundant phospholipids by adding a precursor ion in the negative mode or by using positive ion neutral loss scans. ${ }^{23}$

Studies have shown that the use of methanol as a mobile phase for chromatographic separation provided significant advantages over acetonitrile, because elution of all GPCho occurred in a very narrow time window and their retention behavior on reversed-phase columns could be predicted and decreased by increasing the percentage of the organic phase. ${ }^{24}$ The PPL tended to elute at a high content of the organic mobile phase $^{25}$ and were completely removed from the system at the end of a run by flushing the analytical column with isopropanol. $^{26}$

The behavior of PPL has also been investigated on hydrophobic interaction liquid chromatography (HILIC) columns: $:^{27}$ the compounds were focused into 2 groups of peaks (phosphatidyl cholines and lyso-phosphatidyl cholines) and eluted completely from the column in a one gradient cycle. In comparison, on a reversed-phase material, a strong carry-over was observed from one gradient cycle to another. ${ }^{27}$

In some cases, where retention times of target analytes and PPL overlapped, elution of the target substance could be shifted after adding mobile phase modifiers. ${ }^{27}$

\section{Internal standards}

The use of isotope-labeled internal standards can help overcome most of the matrix effects during sample preparation and LC-MS/MS analysis. However, in some cases the internal standard cannot completely fulfill its purpose, because of slight differences in the chemical behavior of the target analyte and internal standard. For example, particular attention has to be paid to analytes showing strong protein binding. ${ }^{28}$ Generally it is necessary to allow enough time for the internal standard to properly equilibrate and bind to the protein before extraction, to ensure identical behavior of the internal standard and target analyte. ${ }^{29} \mathrm{~A}$ method has been described to determine the extent of protein binding of corticosteroids. ${ }^{30}$ In theory, this method could be extended to other substances and be used to compare the protein-affinity of an analyte and its internal standard. It is important that the release of analytes from the protein (e.g. by adding organic solvents for protein precipitation, $o$-phosphoric acid for breakdown of non-covalent intermolecular interactions $^{31}$ or dithiothreitol (DTT) or tris(2-carboxyethyl)phosphine (TCEP) for reduction of disulfide bonds) has the same impact on the analyte and isotope-labeled standard. A case was reported, where the higher susceptibility of the internal standard for matrix effects than the target analyte led to an underestimation of up to $50 \%$ in the presence of specific buffers used for SPE sample preparation (Fig. 2). ${ }^{19}$

Generally, ${ }^{13} \mathrm{C},{ }^{15} \mathrm{~N}$ or ${ }^{18} \mathrm{O}$-labeled internal standards are preferable to deuterium labeled analogs ${ }^{32}$ because slight differences of physicochemical properties between hydrogen and deuterium can result in small shifts of retention times of the analyte and internal standard. In some cases, this has led to a different degree of ion suppression for the analyte and the internal standard, resulting in changed analyte/internal standard peak area ratios. ${ }^{33,34}$ Also, deuterium-hydrogen backexchange can occur, which has led to false positive results. ${ }^{35}$ Unfortunately, in many cases only deuterated compounds are commercially available, which increases the need to carefully investigate the stability of the reference standards and the influence of matrix effects on the method.

\section{Optimization of established sample preparation methods}

Even though there has been some recent interest in quantitative analysis of pharmaceutical compounds from biological samples using ambient, direct mass spectrometry techniques such as desorption electrospray ionization (DESI) or direct analysis in real time (DART), with little or no prior sample preparation or chromatography, ${ }^{36}$ sample clean-up remains a critical step in most LC-MS analyses of small molecules in biofluids.

\section{Protein precipitation}

The simplest sample preparation approach for biofluids is protein removal. Proteins can be denatured using acids or heat, or removed by using ultrafiltration cut-off membranes. ${ }^{37}$ Another possibility is to use organic solvents for protein precipitation (PPT). PPT removes a part of the phospholipid content present in serum and plasma samples, depending on the organic solvent used. Studies have shown that methanol extracts contain $40 \%$ more phospholipids compared to acetonitrile, ${ }^{11}$ and are also less clean than tetrahydrofuran or ethanol extracts. ${ }^{38}$

\section{Solid-phase extraction (SPE)}

Silica-based sorbents in SPE cartridges have excellent retention capacity for PPL when eluted with $100 \%$ acetonitrile. ${ }^{39}$ Clean extracts were also obtained by including a washing step with up to $50 \%$ methanol, but this strongly affected the recovery of polar analytes. $^{39}$ Large amounts of methanol eluted significant amounts of phospholipids from silica-based reversed-phase SPE cartridges. Methanol contents of 60,70 and $80 \%$ for elution of samples on phenyl, C8 and C18 phases resulted in a high concentration of phospholipids in the extracts. Acetonitrile appeared to be a stronger eluent for phospholipids on reversedphase materials when present at levels up to $50 \%$. The same study showed that the recovery of lysophosphatidylcholines decreased with the increasing content of acetonitrile (>50\%), reaching its minimum at a $100 \%$ organic phase. ${ }^{40}$ The retention 


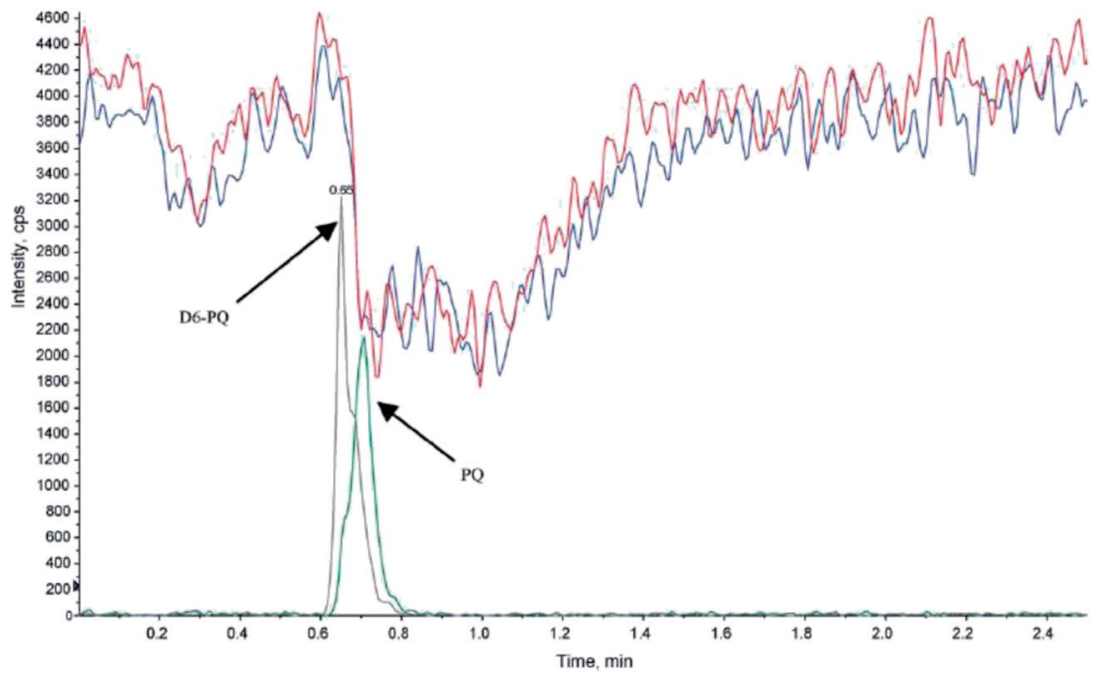

Fig. 2 Injection of extracted blank human plasma ( $+0.2 \mu \mathrm{L}$ triethylamine, blue and red traces) with an overlay of the control sample (20 $\mathrm{ng} \mathrm{mL}$, grey and green traces) containing piperaquine $(P Q)$ and internal standard $\left(d_{6}-P Q\right)$ during post-column infusion at $10 \mu \mathrm{L}$ min ${ }^{-1}$ of $P Q$ and $d_{6}-P Q$ $\left(1.2 \mathrm{ng} \mathrm{mL}^{-1}\right.$ ). Electrospray ionization of the analytes was performed in positive ion mode; the MRM transitions were $\mathrm{m} / \mathrm{z} 535 \rightarrow 288$ and $\mathrm{m} / \mathrm{z}$ $541 \rightarrow 294$ for $P Q$ and D6-PQ, respectively (reprinted with permission from ref. 19).

of phospholipids on the sorbent increased by interactions with residual silanol groups, as was shown by comparison of endcapped and non-endcapped materials. Silica-based sorbents were compared to polymeric phases regarding extraction of phospholipids, and the tested materials showed comparable efficiency. ${ }^{\mathbf{4 0}}$

Studies comparing different sample preparation methods in terms of matrix effects and analyte recovery demonstrated that

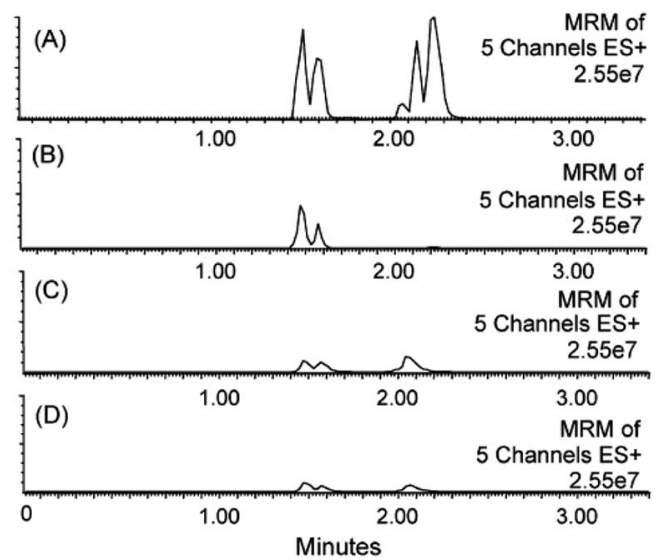

Fig. 3 MRM traces for five residual phospholipids in rat plasma extracts after sample preparation by (A) acetonitrile PPT, (B) reversedphase polymeric SPE, (C) silica-based pure cation exchange, and (D) mixed-mode cation exchange SPE. The phospholipids monitored were 1-palmitoyl-2-hydroxy-sn-glycero-3-phosphocholine $(\mathrm{m} / \mathrm{z}$ 496.35), 1-stearoyl-2-hydroxy-sn-glycero-3-phosphocholine $(\mathrm{m} / \mathrm{z}$ 524.37), 1-hexadecanoyl-2-(9Z,12Z-octadecadienoyl)-sn-glycero-3phosphocholine ( $\mathrm{m} / \mathrm{z}$ 758.57), 1-(9Z,12Z-octadecadienoyl)-2$(5 Z, 8 Z, 11 Z, 14 Z$-eicosatetraenoyl)-sn-glycero-3-phosphocholine $(\mathrm{m} / \mathrm{z}$ 806.57) and a fifth glycerophosphocholine lipid of molecular weight 703.57 Da. MRM analysis was performed on an ESI triple quadrupole LC-MS/MS system using a methanol-water gradient at $\mathrm{pH} 10$ (reprinted with permission from ref. 11). mixed-mode strong anion exchange SPE was more effective than PPT and LLE for polar and non-polar analytes in plasma (Fig. 3). ${ }^{11,41}$

HILIC-SPE was evaluated as an effective method to remove phospholipids from serum and plasma samples. ${ }^{26}$ The retention of phospholipids was shown to increase when samples were diluted with acetone. For some applications to urine samples, HILIC materials were more effective than reversedphase materials. ${ }^{42}$ The polar metabolites in urine had to be separated from the salts and other polar components present in urine. Orthogonal separation using both HILIC and reversedphase materials for sample preparation and chromatography improved the effectiveness of sample clean-up. ${ }^{42}$

Overall, SPE has a very broad range of applications in the LCMS/MS quantification of small molecules in biofluids. ${ }^{43-47}$

\section{Liquid-liquid extraction (LLE)}

Liquid-liquid extraction has found numerous applications for analysis of pharmaceuticals and their metabolites. The concentration of residual phospholipids in the extract is usually lower compared to other techniques such as mixed-mode SPE; on the other hand, the extraction efficiency for highly polar analytes is also lower. ${ }^{29}$ The choice of extraction solvent is very important to reduce unspecific extraction of matrix components. ${ }^{41}$ Halogenated solvents such as chloroform or dichloromethane $\mathrm{es-50}^{\mathbf{8}}$ are commonly used in combination with hydrophilic solvents (e.g. alcohols) for extraction of polar compounds; they also have high affinity for lipids. ${ }^{38}$

As non-ionized analytes are more efficiently extracted by organic solvents than charged species, particular attention has to be paid to the $\mathrm{pH}$ of the sample prior to LLE. As a general rule, the $\mathrm{pH}$ should be between $\mathrm{p} K_{\mathrm{a}}$ and $\left(\mathrm{p} K_{\mathrm{a}}-2\right)$ for acidic analytes and between $\mathrm{p} K_{\mathrm{a}}$ and $\left(\mathrm{p} K_{\mathrm{a}}+2\right)$ for basic analytes, ${ }^{51}$ to increase the extraction recovery. This obviously applies only if 
the stability of the main analyte and its potentially labile metabolites is given in this $\mathrm{pH}$ range. ${ }^{29}$

Extraction using methyl-tert-butylether (MTBE) has shown good results, ${ }^{52}$ but significantly lower analyte recoveries were seen compared to mixed mode SPE and PPT, especially for polar analytes. ${ }^{\mathbf{1 1}}$ Only traces of phospholipids were found in MTBE and $n$-butylchloride extracts of serum and plasma samples. ${ }^{53}$ However, particular attention has to be paid to the process, when several sample preparation steps are combined. The clean extracts obtained with MTBE for untreated serum or plasma can show a high recovery for phospholipids if the samples contain a high percentage of acetonitrile, e.g. after protein precipitation (Fig. 4). ${ }^{53}$

Extraction time also plays an important role for the specific extraction of target analytes compared to matrix components. A study showed that a 5 min extraction time yielded a cleaner extract and better recovery for the target compound than 20 min, indicating that matrix compounds diffuse slower into the extraction solvent. ${ }^{52}$

To improve low recovery rates of LLE for strongly hydrophilic compounds, extraction procedures using water miscible solvents have been considered. Complex methods were reported in the past that use temperatures below $0{ }^{\circ} \mathrm{C}$ to achieve phase separation of serum samples and extraction solvent. ${ }^{54} \mathrm{~A}$ more convenient way to achieve phase separation between an aqueous sample and a water-miscible solvent is salt-assisted liquid-liquid extraction (SALLE), where the polarity of the aqueous phase is increased by adding high concentration of salt, leading to phase separation. ${ }^{55}$ This approach has been used for quantitation of pharmaceutical compounds from biofluids using $\mathrm{LC}^{56}$ or LC-MS/MS. ${ }^{57-59}$

\section{Novel sample preparation methods}

Many common interferents can be removed with conventional sample preparation methods (e.g. protein precipitation, SPE, and LLE), but optimization of these techniques for specific applications is often complex, time-consuming and frequently involves multiple steps. Many common interferents can be removed with conventional sample preparation methods (e.g. protein precipitation, SPE, and LLE), but optimization of these techniques for specific applications is often complex, timeconsuming and frequently involves multiple steps. Moreover, some challenges involving very small sample volumes and low abundant analytes remain. If repeated analyses are required from the same sample and if no further sampling is possible, sample preparation sometimes has to be performed using a sample volume as low as a few microliters. Similar difficulties apply to assays for metabolites or biomarkers that are present at very low concentration levels in human samples. Here, the method must be able to pre-concentrate the target substance(s), additionally to removing all other components of the matrix. New developments for sample preparation methods are therefore often directed towards simplification and possible automation, miniaturization and specificity enhancements of the clean-up process. New developments for sample preparation methods are therefore often directed towards simplification and possible automation, miniaturization and specificity enhancements of the clean-up process. In the following, the most promising recent developments are briefly summarized.

\section{Supported liquid extraction (SLE)}

Even though LLE is mostly a very effective sample preparation method, it has limitations, in particular low sample throughput. Several extractions are often required to improve analyte recovery, sample handling is labor-intensive and timeconsuming, and emulsions can form at the interface between liquid layers. These limitations can be overcome by using supported liquid extraction (SLE), where aqueous samples are adsorbed on a porous solid support material, e.g. diatomaceous earth. Some studies have shown analyte recovery from SLE that was comparable or higher than LLE. ${ }^{60}$

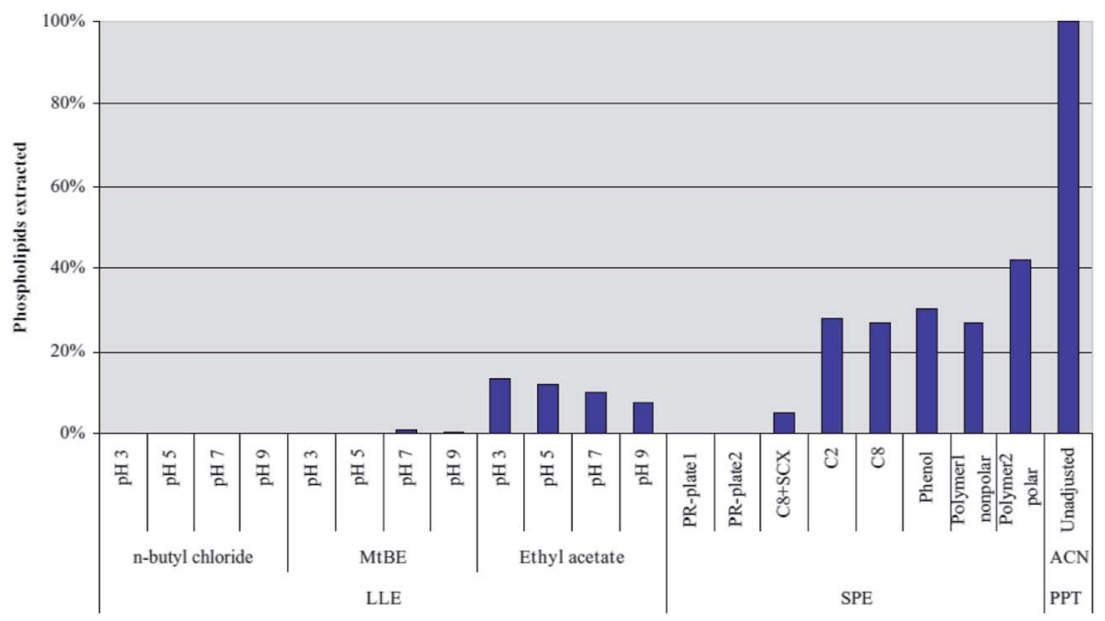

Fig. 4 Extraction of C16:0 lysophosphatidylcholine (C16:0 lyso-PC) from human plasma using liquid-liquid extraction with three different solvents at different $\mathrm{pH}$ values. Comparison to solid-phase extraction and two commercial phospholipid removal sorbents (PR-plate 1 and PRplate 2). Lyso-PC was monitored using the following MRM transition: $m / z 496 \rightarrow 184$ (reprinted with permission from ref. 53). 
SLE has been shown to effectively remove the majority of phospholipids when the extraction conditions were carefully optimized. ${ }^{61}$ The efficiency of several extraction solvents was also compared for SLE: ${ }^{7}$ ethyl acetate removed about 85\%, MTBE removed more than $99 \%$ of total phospholipids. Dichloromethane removed $99.5 \%$ of the phospholipids when used alone; its removal efficiency decreased to $95 \%$ when isopropanol was added. However, addition of water-soluble solvents to the samples (e.g., acetonitrile or methanol) prior to SLE extraction led to higher matrix effects for some analytes. ${ }^{7}$ Isopropanol combined with dichloromethane also yielded low concentrations of phospholipids in the extract. ${ }^{7}$

The SLE technique has been implemented in various LC-MS/ MS methods recently. ${ }^{62-67}$ It was particularly powerful for normal phase separation systems, since the high percentage of organic solvent in the eluate did not need to be evaporated prior to injection into the LC-MS/MS system. ${ }^{68}$

\section{Phospholipid removal plates}

The use of hybrid precipitation/SPE plates for selective removal of phospholipids and precipitated proteins has been increasing over the past few years. ${ }^{\mathbf{4 3 8 , 6 9 , 7 0}}$ Several types of these plates are now commercially available, e.g. Hybrid SPE ${ }^{\mathrm{TM}}$ (Sigma Aldrich), Ostro $^{\mathrm{TM}}$ (Waters), Captiva ${ }^{\mathrm{TM}}$ ND (Agilent) and Phree ${ }^{\mathrm{TM}}$ (Phenomenex). These plates have shown very effective extractions of phospholipids compared to PPT. ${ }^{71}$ For example, the Hybrid SPE plate specifically retains phospholipids by Lewis acid-base interactions between zirconia ions - which are bonded to the stationary phase - and the phosphate group of the phospholipids. Acetonitrile with $1 \%$ formic acid is used as the precipitation agent; formic acid has important influence on the recovery of the analytes. ${ }^{72}$ Hybrid SPE extracts have shown to contain significantly lower phospholipid concentrations as compared to PPT. ${ }^{4}$ Ostro uses a combination of protein precipitation and extraction on a C18 sorbent. Several applications using these products have been reported. ${ }^{73-75}$

Other approaches are also possible for removal of phospholipids. A study showed that addition of a colloidal silica suspension together with lanthanum chloride to plasma samples resulted in a reproducible sample clean-up without loss of the analyte of interest. ${ }^{76}$

\section{Magnetic beads}

Magnetic particles and nanoparticles (MNPs) are becoming increasingly interesting for sample preparation. They have been used for extraction and pre-concentration of drugs in complex biological fluids. ${ }^{77-79}$ They consist of a magnetic core $\left(e . g . \mathrm{Fe}_{3} \mathrm{O}_{4}\right)$ coated with a polymer material, to which specific functionalities can be added (Fig. 5). ${ }^{80}$ Sample preparation steps are similar to SPE (loading, washing and elution). The magnetic particles suspended in solution can be handled as a liquid. Obviously, the big advantage of magnetic beads is that after sample extraction, the beads are pulled to the tube wall, the supernatant is removed and the wall-bound beads washed with an appropriate solvent. The loaded beads are then re-suspended.
The entire procedure is fast and simple, and complete automation is readily possible.

Several applications have been reported, where either analytes are selectively extracted from a complex matrix ${ }^{81-83}$ or where the matrix components were removed from the sample, leaving a clean extract behind that can be directly injected into the LC-MS system. ${ }^{\mathbf{8 4}}$ Using matrix-assisted laser desorption/ ionization (MALDI), the analytes can also be analyzed without having to be eluted from the magnetic beads first. ${ }^{85}$ The possible modifications on the surface of the magnetic beads are similar to conventional SPE and involve hydrophobic coatings, ion exchange functionalities, molecular imprinted polymers (MIP) ${ }^{86}$ restricted access ${ }^{\mathbf{8 7}}$ or affinity materials. ${ }^{88}$ Magnetic particles have also been coated with carbon nanotubes and used to extract aromatic compounds. ${ }^{89}$

\section{Turboflow}

Turboflow extraction is usually carried out online before chromatographic separation and uses columns with large particle sizes in conjunction with high flow rates. ${ }^{90}$ Samples can be directly injected after dilution; sometimes a protein precipitation step is required before injection. The target analytes are retained in the pores of the column, whereas matrix components are flushed through and discarded directly to waste. The analytes are then eluted from the trapping column using organic solvents. This method has the advantage of fast and generic method development but unfortunately it can also show high carry-over effects. ${ }^{91}$ A study reported that this technique had no significant impact on phospholipid removal from serum and plasma samples, and still needed extensive chromatographic separation after clean-up to avoid matrix effects. ${ }^{92}$ Other groups reported successful applications for quantification of various substances (drugs, steroids, phenolic compounds, etc. $)^{\mathbf{9 3 - 1 0 2}}$ in human serum, urine and dried blood spots using reversed-phase, ion exchange or mixed-mode materials.

\section{Monolithic spin column extraction}

Monolithic spin column extraction is a fast sample preparation method that uses a spin column packed with octadecyl silanebonded monolithic silica as the extraction device. ${ }^{\mathbf{1 0 3}}$ The sample is loaded onto the sorbent by centrifugation; the same procedure is performed for washing and elution steps. ${ }^{\mathbf{1 0 4}}$ This technique is fast and easy, requires only small amounts of solvents and allows high sample throughput. Unfortunately, the method can only be applied over a limited $\mathrm{pH}$ range because of possible degradation of the monolithic silica phase. ${ }^{\mathbf{1 0 4}}$ Several applications have been reported for quantification of various analytes from human samples, using underivatized, ${ }^{105} \mathrm{C} 18,{ }^{106-108}$ ion exchange $^{109}$ or mixed-mode phases (C-C18, TiO-C18, C18-ion exchange). ${ }^{110-112}$

\section{Microextraction by packed sorbent (MEPS)}

This recent sample preparation technique is based on the miniaturization of conventional SPE, using a gas-tight syringe as extraction device. The method is designed for sample volumes from 10 to $1000 \mu \mathrm{L}$ and can be connected online to 


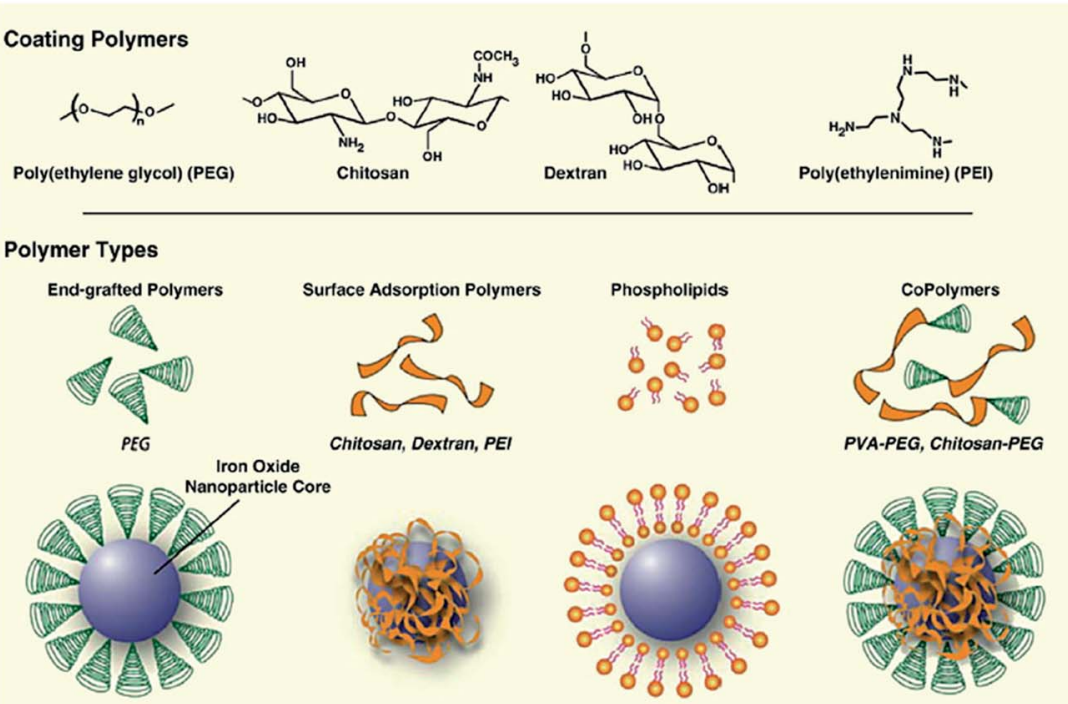

Fig. 5 Assembly of polymers onto the surface of magnetic nanoparticle cores (reprinted with permission from ref. 168).

LC-MS or GC-MS. Compared to conventional SPE, MEPS is easy to use, faster and needs significantly lower amounts of organic solvents. Additionally, MEPS sorbents can be used for up to 100 extractions. ${ }^{113}$

Packing materials for MEPS are similar to sorbents used for SPE. Essentially, any sorbent material and functionalization can be applied. For example, silica-based materials (C2, C8, C18), ${ }^{114-118}$ with additional ion exchange functionality ${ }^{119}$ or even as mixed-mode materials, ${ }^{120}$ restricted access materials (RAM), HILIC, carbon, polystyrene-divinylbenzene copolymers (PSDVB) or molecular imprinted polymers ${ }^{\mathbf{1 1 3 , 1 2 1}}$ have been utilized for MEPS.

The method has been implemented in several recent reports for quantification of pharmaceutical compounds from human biological samples (urine, plasma, oral fluid and whole blood), including antipsychotic drugs, ${ }^{\mathbf{1 1 9}}$ cardiac drugs, ${ }^{\mathbf{1 1 4}}$ local anesthetics, ${ }^{\mathbf{1 1 5}, \mathbf{1 2 1}}$ phenolic acid, ${ }^{\mathbf{1 1 6}}$ immunosuppressants, ${ }^{\mathbf{1 1 7}}$ opioids $^{120}$ and antidepressants. ${ }^{118}$ Recent studies have also reported the successful extraction of trazodone from plasma with polymer nano-fibers as the extraction sorbent. ${ }^{\mathbf{1 2 2}}$

\section{Carbon nanotubes}

Carbon surfaces have the ability to retain substances by strong hydrophobic interactions. These materials are therefore interesting for reversed-phase extractions of hydrophilic substances. Carbon nanotubes (CNTs) are hollow cylinders that consist of one (single-wall carbon nanotubes, SWCNTs) or several (multiwalled carbon nanotubes, MWCNTs) graphene layers. ${ }^{\mathbf{1 2 3}}$ Because of their large surface areas, CNTs have a high adsorption capacity. They show high affinity towards aromatic compounds that can be adsorbed via $\pi-\pi$ interactions. ${ }^{\mathbf{1 2 4}}$ CNTs can be packed into SPE cartridges or used for dispersive solid phase extraction. ${ }^{\mathbf{1 2 5 - 1 2 7}}$ Common target analytes are small, hydrophobic molecules extracted from water samples. Very few applications to biofluids have been reported so far. A method for quantitation of diuretics from urine ${ }^{128}$ has been published as well as plasma peptide analysis. ${ }^{129}$ The specificity of the extraction can be enhanced by derivatizing the surface of CNTs with functional groups. A method was recently shown for the determination of anti-inflammatory drugs from urine using carboxylated CNTs for sample clean-up. ${ }^{130}$ To further improve both specificity and handling of the sample clean-up, magnetic CNTs coated with molecular imprinted polymers have been synthetized and used for extraction of BSA from serum samples. $^{\mathbf{1 3 1}}$

\section{Restricted access materials (RAM)}

Restricted access materials allow extractive clean-up of biofluids by utilizing physical and chemical diffusion barriers. RAM consist of a porous material with a restrictive and hydrophilic outer surface that prevents retention of large interfering molecules such as proteins and phospholipids, combined with smaller inner pores with hydrophobic surfaces that only molecules with low molecular weight can reach. ${ }^{\mathbf{1 3 2}}$ This technique is commonly used for online sample clean-up, with the advantage that samples dissolved in almost any solvent can be loaded, even MS incompatible solvents, before elution with the mobile phase used for chromatographic separation. There are two types of RAM phases: ${ }^{133}$ internal surface phase (ISP) materials use size exclusion to prevent the matrix components from reaching the inner layer; semi-permeable surface (SPS) materials chemically exclude matrix components by polymeric- or protein coating of the outer layer. In both cases, the inner layer can be functionalized to enhance the specificity of the method. ${ }^{\mathbf{1 3 4}}$ Molecular imprinted polymers are a special form of restricted access materials; they are discussed below.

Application of sample clean-up using RAM includes quantification of antimicrobial agents, immunosuppressants etc. from human biological samples prior to LC-MS/MS analysis. ${ }^{\mathbf{1 3 5 , 1 3 6}}$ RAM have also been used in combination with magnetic 
particles to quantify therapeutic drugs and steroids from biofluids. ${ }^{87,137-140}$ An application was published that reported the synthesis of chiral RAM materials for extraction of enantiomeric drugs from plasma samples. ${ }^{141}$

\section{Immunosorbents}

Immunosorbents use the principle of antigen-antibody affinity for highly specific retention of target substances. The desired antibody is bound to a solid support or gel, which can be used as SPE or micro-SPE sorbent, MEPS or in columns. ${ }^{123}$ The target analytes can be specifically extracted from complex matrices, which allows thorough sample clean-up prior to instrumental analysis. A study has shown that the capacity of monoclonal antibodies was significantly higher than that of polyclonal antibodies. ${ }^{142}$ This technique has been used as in-tube SPME to quantify interferon $\alpha$ from plasma samples ${ }^{143}$ as well as SPE extraction of ProGRP ${ }^{144}$ and ochratoxin ${ }^{145}$ from serum. Sample preparation techniques with high specificity towards the target analyte are required if the target analyte is present at very low concentration levels or in cases where structurally similar interferents (e.g. isobars) influence the analysis. ${ }^{146}$ The immunosorbent extraction usually involves high costs, however, and also requires host animals to grow the required antibodies. Sometimes, the antibodies can be replaced by synthetic alternatives of comparable specificity, such as molecular imprinted polymers or aptamers (see below).

\section{Molecular imprinted polymers (MIPs)}

MIPs use the principle of affinity chromatography to maximize the specificity for the analyte(s) of interest. The target analyte or a structurally-related compound is used as a template for the synthesis of the MIP by copolymerization of a complex formed by the template and a functional monomer. The template molecule is then removed, leaving a rigid three-dimensional cavity that is complementary to the target analyte. ${ }^{147}$

The synthesis of these adsorbents is often inexpensive and has shown to be fast and reproducible; the materials also have high capacity and can be regenerated and used several times. ${ }^{148}$ The MIP principle enables highly specific extraction of the target and structurally similar compounds (e.g. a drug and its metabolites) from complex matrices, and pre-concentration of the sample. The specificity of this technique has been shown in several applications. For example, a MIP sorbent developed for tylosin was able to differentiate between tylosin and the closely related narbomycin as well as the remotely similar tylactone. (Fig. 6). Both the target analyte and structurally similar compound were quantitatively extracted, whereas the interfering substance did not show any affinity for the sorbent. ${ }^{149}$

MIP can be used in various forms, for online or off-line processes such as molecular imprinted solid phase extraction (MISPE), ${ }^{150}$ magnetic MIP, ${ }^{151,152}$ solid-phase micro-extraction (SPME), needle/micropipette tip, dynamic liquid-liquid-solid micro-extraction (DLLSME) or molecular imprinted stir-bar sorptive extraction (MI-SBSE). ${ }^{153,154}$ This concept has been applied to samples with complex matrices, for example, for benzodiazepines in plasma, ${ }^{155}$ nucleoside reverse transcriptase

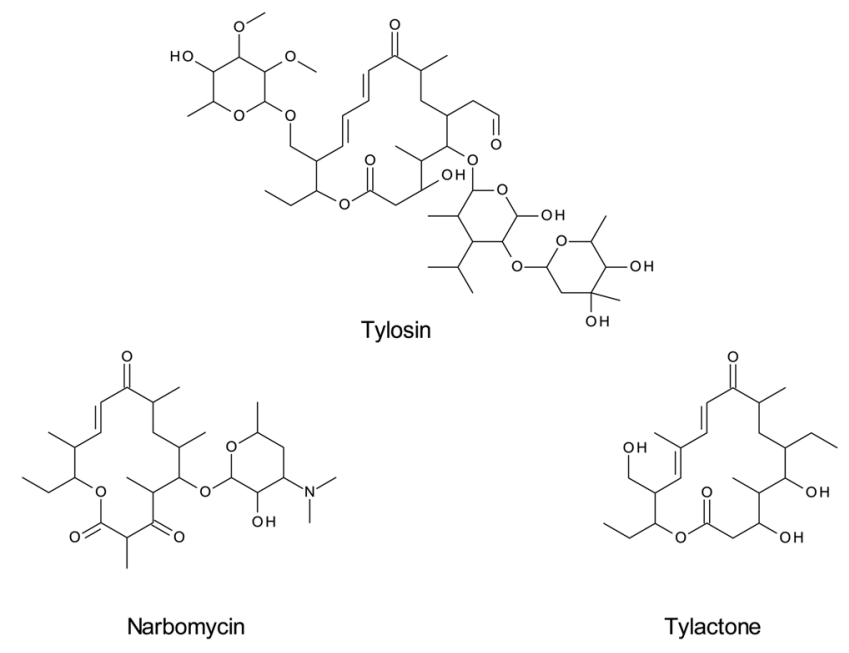

Fig. 6 Structures of tylosin and two structurally-related compounds, narbomycin and tylactone.

inhibitors in serum, ${ }^{156}$ cocaine $^{157}$ or ketamine ${ }^{158}$ from hair extract, testosterone ${ }^{159}$ and tobacco-specific cancer biomarkers ${ }^{160}$ from urine. MIP-coated fibers for solid phase microextraction (SPME) have also been used for extraction of linezolid from human biofluids. ${ }^{161}$ This technique has shown to provide much cleaner extracts than other sample preparation methods such as LLE. ${ }^{155}$ However, this technology still needs some improvement and has several drawbacks, including possible template bleeding, sometimes tedious synthesis procedures, and problematic application to aqueous samples. ${ }^{147,150}$

\section{Aptamers}

Another possibility to increase specificity for the target analyte is the use of aptamers immobilized on a solid sorbent for sample preparation. Aptamers are synthetic single-stranded oligonucleotides capable of binding specific analytes with a high affinity through hydrogen bonding, van der Waals forces and dipole interactions. ${ }^{123,162}$ They are specifically prepared for each target molecule; that is, several nucleic acids have to be tested in vitro for each target. Selected nucleic acids with high affinity for the analytes are isolated and amplified using a process called systematic evolution of ligands by exponential enrichment (SELEX). ${ }^{163}$ The major advantage compared to antibodies is that aptamers can be synthetized directly, without the need for laboratory animals. They can be regenerated within minutes and reused several times. The technique has been used for the selective extraction of cocaine from plasma ${ }^{164,165}$ and for extraction of tetracyclines from biological fluids in combination with ion mobility spectrometry. ${ }^{166}$ The high affinity of a target substance to an extraction sorbent is clearly shown in these applications as well as the importance of the sequence of the oligonucleotides. The sequence is specific for a particular compound and will become inactive if the oligonucleotides are grafted in a randomized order. ${ }^{163}$ Recoveries of up to $90 \%$ confirm the high specificity of this technique, even in complex 
samples such as plasma. ${ }^{\mathbf{1 6 3}}$ Aptamers have also been immobilized on polymeric nano-fibers and extraction of thrombin from serum was shown. ${ }^{167}$

\section{Conclusion}

Common problems encountered during development of an LC-MS/MS assay for the quantification of small molecules from biological samples include loss of sensitivity and specificity due to matrix effects. Sample preparation is therefore an indispensable part of the analytical workflow. The possible influence of matrix effects on LC-MS/MS assays has been extensively studied and several methods have been published to identify and avoid these effects. Considerable progress has been made in the improvement of sample preparation routines in the last few years. New trends are directed towards either increasing the specificity of the extraction for the target analyte or removing as much of the matrix components as possible. Miniaturization and automation of these techniques are on-going efforts, leading to cheaper, more robust and fully automated LC-MS/MS assays that will significantly impact pharmaceutical analyses of biofluids in the future.

\section{List of abbreviations}

$\begin{array}{ll}\text { APCI } & \text { Atmospheric pressure chemical ionization } \\ \text { BSA } & \text { Bovine serum albumin } \\ \text { CNT } & \text { Carbon nanotubes } \\ \text { CSF } & \text { Cerebrospinal fluid } \\ \text { DART } & \text { Direct analysis in real time } \\ \text { DESI } & \text { Desorption electrospray ionization } \\ \text { DLLSME } & \text { Dynamic liquid-liquid-solid microextraction } \\ \text { DTT } & \text { Dithiothreitol } \\ \text { ESI } & \text { Electrospray ionization } \\ \text { GC-MS } & \text { Gas chromatography-mass spectrometry } \\ \text { GPCho } & \text { Glycerophosphocholines } \\ \text { HILIC } & \text { Hydrophobic interaction liquid chromatography } \\ \text { IS-MRM } & \text { In-source multiple reaction monitoring } \\ \text { ISP } & \text { Internal surface phase } \\ \text { LC-MS } & \text { Liquid chromatography-mass spectrometry } \\ \text { LC-MS/ } & \text { Liquid chromatography-tandem mass spectrometry } \\ \text { MS } & \\ \text { LLE } & \text { Liquid-liquid extraction } \\ \text { MALDI } & \text { Matrix-assisted laser desorption/ionization } \\ \text { MEPS } & \text { Microextraction by packed sorbent } \\ \text { MIP } & \text { Molecular imprinted polymers } \\ \text { MI-SBSE } & \text { Molecular imprinted stir-bar sorptive extraction } \\ \text { MISPE } & \text { Molecular imprinted solid phase extraction } \\ \text { MNP } & \text { Magnetic nanoparticles } \\ \text { MRM } & \text { Multiple reaction monitoring } \\ \text { MTBE } & \text { Methyl-tert-butylether } \\ \text { MWCNT } & \text { Multi-walled carbon nanotubes } \\ \text { PPL } & \text { Phospholipids } \\ \text { PPT } & \text { Protein precipitation } \\ \text { PQ } & \text { Piperaquine } \\ \text { PS-DVB } & \text { Polystyrene-divinylbenzene } \\ \text { RAM } & \text { Restricted access materials } \\ & \end{array}$

SALLE SELEX

SLE

SPE

SPME

SPS SWCNT TCEP
Salt-assisted liquid-liquid extraction

Systematic evolution of ligands by exponential enrichment

Supported liquid extraction

Solid-phase extraction

Solid-phase microextraction

Semi-permeable surface

Single-wall carbon nanotubes

Tris(2-carboxyethyl)phosphine

\section{Acknowledgements}

DAV acknowledges research support by the Alfried Krupp von Bohlen und Halbach-Stiftung.

\section{References}

1 Using Mass Spectrometry for Drug Metabolism Studies, ed. W. A. Korfmacher, 2004.

2 Y. Modhave, Int. J. Pharm. Phytopharm. Res., 2012, 1, 403-405.

3 B. K. Matuszewski, M. L. Constanzer and C. M. Chavez-Eng, Anal. Chem., 2003, 75, 3019-3030.

4 V. Pucci, S. Di Palma, A. Alfieri, F. Bonelli and E. Monteagudo, J. Pharm. Biomed. Sci., 2009, 50, 867-871.

5 B. K. Matuszewski, M. L. Constanzer and C. M. Chavez-Eng, Anal. Chem., 1998, 70, 882-889.

6 R. Bonfiglio, R. C. King, T. V. Olah and K. Merkle, Rapid Commun. Mass Spectrom., 1999, 13, 1175-1185.

7 H. Jiang, H. Cao, Y. Zhang and D. M. Fast, J. Chromatogr. B: Anal. Technol. Biomed. Life Sci, 2012, 891, 71-80.

8 W. X. Lv, Talanta, 2010, 80, 1406-1412.

9 L. L. Jessome and D. A. Volmer, LCGC North Am., 2006, 24, 498-510.

10 F. Janusch, L. Kalthoff, G. Hamscher and S. A. Mohring, Bioanalysis, 2013, 5, 2101-2114.

11 E. Chambers, D. M. Wagrowski-Diehl, Z. L. Lu and J. R. Mazzeo, J. Chromatogr. B: Anal. Technol. Biomed. Life Sci, 2007, 852, 22-34.

12 Z. Q. Ye, H. Tsao, H. Gao and C. L. Brummel, Bioanalysis, 2011, 3, 1587-1601.

13 M. Ruggieri, C. Tortorella, E. Ceci, D. Paolicelli, V. Solfrizzi, G. Di Bitonto, C. Pica, M. Mastrapasqua, P. Livrea and M. Trojano, Age Ageing, 2011, 40, 391-395.

14 S. Kowal, P. Balsaa, F. Werres and T. C. Schmidt, Anal. Bioanal. Chem., 2013, 405, 6337-6351.

15 P. Basilicata, N. Miraglia, M. Pieri, A. Acampora, L. Soleo and N. Sannolo, J. Chromatogr. B: Anal. Technol. Biomed. Life Sci, 2005, 818, 293-299.

16 A. G. Frenich, J. L. M. Vidal, J. L. F. Moreno and R. RomeroGonzalez, J. Chromatogr. A, 2009, 1216, 4798-4808.

17 N. C. Hughes, N. Bajaj, J. A. Fan and E. Y. K. Wong, Bioanalysis, 2009, 1, 1057-1066.

18 R. Lucena, P. Moreno, A. Perez-Rico and P. J. Ginel, Vet. J., 1998, 156, 127-131.

19 N. Lindegardh, A. Annerberg, N. J. White and N. P. Day, J. Chromatogr. B: Anal. Technol. Biomed. Life Sci., 2008, 862, 227-236. 
20 J. J. Myher, A. Kuksis and S. Pind, Lipids, 1989, 24, 408-418. 21 V. Gonzalez-Covarrubias, A. Dane, T. Hankemeier and R. J. Vreeken, Metabolomics, 2013, 9, 337-348.

22 J. L. Little, M. F. Wempe and C. M. Buchanan, J. Chromatogr. B: Anal. Technol. Biomed. Life Sci, 2006, 833, 219-230.

23 Y. Q. Xia and M. Jemal, Rapid Commun. Mass Spectrom., 2009, 23, 2125-2138.

24 S. Silvester and L. Smith, Bioanalysis, 2012, 4, 879-895.

25 Z. M. Liang, Bioanalysis, 2012, 4, 1227-1234.

26 T. Van Damme, M. Lachova, F. Lynen, R. Szucs and P. Sandra, Anal. Bioanal. Chem., 2013, 406, 401-407.

27 B. J. Tan, A. Negahban, T. McDonald, Y. Z. Zhang and C. Holliman, Bioanalysis, 2012, 4, 2049-2058.

28 S. L. Zhou, Q. Song, Y. Tang and N. D. Weng, Curr. Pharm. Anal., 2005, 1, 3-14.

29 D. E. Mulvana, Bioanalysis, 2010, 2, 1051-1072.

30 S. Taylor and A. Harker, J. Pharm. Biomed. Sci., 2006, 41, 299-303.

31 S. Tulipani, R. Llorach, M. Urpi-Sarda and C. AndresLacueva, Anal. Chem., 2013, 85, 341-348.

32 S. Wang, M. Cyronak and E. Yang, J. Pharm. Biomed. Sci., 2007, 43, 701-707.

33 C. J. Briscoe, M. R. Stiles and D. S. Hage, J. Pharm. Biomed. Sci., 2007, 44, 484-491.

34 M. Jemal, A. Schuster and D. B. Whigan, Rapid Commun. Mass Spectrom., 2003, 17, 1723-1734.

35 N. W. Davies, J. A. Smith, P. P. Molesworth and J. J. Ross, Rapid Commun. Mass Spectrom., 2010, 24, 1105-1110.

36 S. X. Yu, E. Crawford, J. Tice, B. Musselman and J. T. Wu, Anal. Chem., 2009, 81, 193-202.

37 C. Bylda, R. Thiele, U. Kobold and D. A. Volmer, Drug Test. Anal., 2013, DOI: 10.1002/dta.1527.

38 C. Ferreiro-Vera, F. Priego-Capote and M. D. Luque de Castro, J. Chromatogr. A, 2012, 1240, 21-28.

39 C. Cote, A. Bergeron, J. N. Mess, M. Furtado and F. Garofolo, Bioanalysis, 2009, 1, 1243-1257.

40 M. Lahaie, J. N. Mess, M. Furtado and F. Garofolo, Bioanalysis, 2010, 2, 1011-1021.

41 S. G. Chinmoy Ghosh, C. P. Shinde and B. Chakraborty, J. Bioequivalence Bioavailability, 2011, 3, 122-127.

42 W. Y. Jian, R. W. Edom, Y. D. Xu and N. D. Weng, J. Sep. Sci., 2010, 33, 681-697.

43 C. Bylda, A. Leinenbach, R. Thiele, U. Kobold and D. A. Volmer, Drug Test. Anal., 2012, 4, 668-674.

44 D. Gode, M. M. Martin, F. Steiner, C. G. Huber and D. A. Volmer, Anal. Bioanal. Chem., 2012, 404, 433-445.

45 R. N. X. Xu, L. M. Fan, M. J. Rieser and T. A. El-Shourbagy, J. Pharm. Biomed. Sci., 2007, 44, 342-355.

46 N. Nakov, K. Mladenovska, N. Labacevski, A. Dimovski, R. Petkovska, A. Dimitrovska and Z. Kavrakovski, Biomed. Chromatogr., 2013, 27, 1540-1546.

47 Z. Leon, A. Chisvert, I. Tarazona and A. Salvador, Anal. Bioanal. Chem., 2010, 398, 831-843.

48 E. L. Oiestad, U. Johansen, M. Stokke Opdal, S. Bergan and A. S. Christophersen, J. Anal. Toxicol., 2009, 33, 372378.
49 J. Ni, H. Ouyang, M. Aiello, C. Seto, L. Borbridge, T. Sakuma, R. Ellis, D. Welty and A. Acheampong, Pharm. Res., 2008, 25, 1572-1582.

50 A. Tracqui, P. Kintz, B. Ludes and P. Mangin, J. Chromatogr. B: Biomed. Sci. Appl., 1997, 692, 101-109.

51 G. Hendriks, D. R. A. Uges and J. P. Franke, J. Chromatogr. B: Anal. Technol. Biomed. Life Sci, 2007, 853, 234-241.

52 A. F. Aubry, Bioanalysis, 2011, 3, 1819-1825.

53 M. Jemal, Z. Ouyang and Y. Q. Xia, Biomed. Chromatogr., 2010, 24, 2-19.

54 M. Yoshida and A. Akane, Anal. Chem., 1999, 71, 1918-1921.

55 I. M. Valente, L. M. Goncalves and J. A. Rodrigues, J. Chromatogr. A, 2013, 1308, 58-62.

56 M. Gupta, A. Jain and K. K. Verma, J. Sep. Sci., 2010, 33, 3774-3780.

57 J. Zhang, H. Q. Wu, E. Kim and T. A. El-Shourbagy, Biomed. Chromatogr., 2009, 23, 419-425.

58 H. Q. Wu, J. Zhang, K. Norem and T. A. El-Shourbagy, J. Pharm. Biomed. Sci., 2008, 48, 1243-1248.

59 P. L. Kole, G. Venkatesh, J. Kotecha and R. Sheshala, Biomed. Chromatogr., 2011, 25, 199-217.

60 Y. Zhang, H. Cao and H. Jiang, Bioanalysis, 2013, 5, 285288.

61 O. A. Ismaiel, T. Y. Zhang, R. G. Jenkins and H. T. Karnes, J. Chromatogr. B: Anal. Technol. Biomed. Life Sci, 2010, 878, 3303-3316.

62 L. J. Owen, F. C. Wu and B. G. Keevil, Ann. Clin. Biochem., 2013, DOI: $10.1177 / 0004563213501478$.

63 M. T. Furlong, B. He, W. Mylott, S. Zhao, T. Mariannino, J. Shen and B. Stouffer, J. Pharm. Biomed. Sci., 2012, 70, 574-579.

64 C. Svanstrom, G. P. Hansson, L. D. Svensson and C. J. Sennbro, J. Pharm. Biomed. Sci., 2012, 58, 71-77.

65 A. B. Biotage, $L C \cdot G C$ Eur., 2012, 12.

66 F. K. Victor and E. Vandell, $L C-G C, 2012$, 76, Special issues.

67 K. G. P. R. Nageswara Rao, K. V. Sravan Kumarb and B. Ramesh, Anal. Methods, 2013, 5, 6693-6699.

68 X. J. a. Y.-L. C. Jiongwei Pan, Pharmaceutics, 2010, 2, 105118.

69 M. Moriarty, A. Lee, B. O'Connell, M. Lehane, H. Keeley and A. Furey, Chromatographia, 2012, 75, 1257-1269.

70 H. Jiang, Y. Zhang, M. Ida, A. LaFayette and D. M. Fast, J. Chromatogr. B: Anal. Technol. Biomed. Life Sci., 2011, 879, 2162-2170.

71 D. Neville, R. Houghton and S. Garrett, Bioanalysis, 2012, 4, 795-807.

72 S. Ahmad, H. Kalra, A. Gupta, B. Raut, A. Hussain and M. A. Rahman, J. Pharm. BioAllied Sci., 2012, 4, 267-275.

73 J. M. Cunliffe, J. X. Shen, X. R. Wei, D. P. Dreyer, R. N. Hayes and R. P. Clement, Bioanalysis, 2011, 3, 735-743.

74 L. Ye, J. Shi, S. Wan, X. Yang, Y. Wang, J. Zhang, D. Zheng and Z. Liu, Biomed. Chromatogr., 2013, 27, 1532-1539.

75 K. Ardjomand-Woelkart, M. Kollroser, L. Li, H. Derendorf, V. Butterweck and R. Bauer, Anal. Bioanal. Chem., 2011, 400, 2371-2381.

76 S. T. Wu, D. Schoener and M. Jemal, Rapid Commun. Mass Spectrom., 2008, 22, 2873-2881. 
77 I. I. Sukhanova, M. A. Dikunets, E. D. Viryus and G. M. Rodchenkov, J. Anal. Chem., 2011, 66, 807-814.

78 J. F. Peter and A. M. Otto, Proteomics, 2010, 10, 628-633.

79 M. Tsunehiro, Y. Meki, K. Matsuoka, E. Kinoshita-Kikuta, E. Kinoshita and T. Koike, J. Chromatogr. B: Anal. Technol. Biomed. Life Sci., 2013, 925, 86-94.

80 Z. Karimi, L. Karimi and H. Shokrollahi, Mater. Sci. Eng., C, 2013, 33, 2465-2475.

81 A. A. Rajabi, Y. Yamini, M. Faraji and S. Seidi, Med. Chem. Res., 2013, 22, 1570-1577.

82 K. Konig and M. Vogeser, Eur. J. Mass Spectrom., 2012, 18, 413-417.

83 M. Vogeser, A. Geiger, R. Herrmann and U. Kobold, Clin. Biochem., 2009, 42, 915-918.

84 K. Konig, S. F. Goethel, V. M. Rusu and M. Vogeser, Clin. Biochem., 2013, 46, 652-655.

85 D. Gode and D. A. Volmer, Rapid Commun. Mass Spectrom., 2013, 27, 1011-1018.

86 X. Liu, J. Liu, Y. Huang, R. Zhao, G. Liu and Y. Chen, J. Chromatogr. A, 2009, 1216, 7533-7538.

87 Y. Wang, Y. X. Wang, L. Chen and Q. H. Wan, J. Magn. Magn. Mater., 2012, 324, 410-417.

88 Y. Tao, Z. Chen, Y. Zhang, Y. Wang and Y. Cheng, J. Pharm. Biomed. Sci., 2013, 78-79, 190-201.

89 N. Rastkari and R. Ahmadkhaniha, J. Chromatogr. A, 2013, 1286, 22-28.

90 L. Couchman, Biomed. Chromatogr., 2012, 26, 892-905.

91 W. M. Mullett, J. Biochem. Biophys. Methods, 2007, 70, 263273.

92 L. H. Du and R. L. White, Rapid Commun. Mass Spectrom., 2008, 22, 3362-3370.

93 E. Abe, F. Ricard, F. Darrouzain and J. C. Alvarez, Anal. Bioanal. Chem., 2013, 405, 239-245.

94 H. Frederiksen, L. Aksglaede, K. Sorensen, O. Nielsen, K. M. Main, N. E. Skakkebaek, A. Juul and A. M. Andersson, Int. J. Hyg. Environ. Health, 2013, 216, 710-720.

95 T. Soeborg, H. Frederiksen, P. Fruekilde, T. H. Johannsen, A. Juul and A. M. Andersson, Clin. Chim. Acta, 2013, 419, 95-101.

96 T. H. Lassen, H. Frederiksen, T. K. Jensen, J. H. Petersen, K. M. Main, N. E. Skakkebaek, N. Jorgensen, S. K. Kranich and A. M. Andersson, Environ. Res., 2013, 126, 164-170.

97 L. Couchman, M. Birch, R. Ireland, A. Corrigan, S. Wickramasinghe, D. Josephs, J. Spicer and R. J. Flanagan, Anal. Bioanal. Chem., 2012, 403, 1685-1695.

98 L. Couchman, S. L. Buckner, P. E. Morgan, M. M. Ceesay, A. Pagliuca and R. J. Flanagan, Anal. Bioanal. Chem., 2012, 404, 513-523.

99 X. He and M. Kozak, Anal. Bioanal. Chem., 2012, 402, 30033010.

100 T. Bernsmann, P. Furst and M. Godula, Food Addit. Contam., Part A, 2011, 28, 1352-1363.

101 T. F. Metz, T. P. Mechtler, J. J. Orsini, M. Martin, B. Shushan, J. L. Herman, R. Ratschmann, C. B. Item, B. Streubel, K. R. Herkner and D. C. Kasper, Clin. Chem., 2011, 57, 1286-1294.
102 R. Harlan, W. Clarke, J. M. Di Bussolo, M. Kozak, J. Straseski and D. L. Meany, Clin. Chim. Acta, 2010, 411, 1728-1734.

103 A. Namera, M. Nagao, A. Nakamoto, S. Miyazaki and T. Saito, J. AOAC Int., 2011, 94, 765-774.

104 X. J. Huang and D. X. Yuan, Crit. Rev. Anal. Chem., 2012, 42, 38-49.

105 A. Nakamoto, M. Nishida, T. Saito, I. Kishiyama, S. Miyazaki, K. Murakami, M. Nagao and A. Namura, Anal. Chim. Acta, 2010, 661, 42-46.

106 T. Saito, T. Fukushima, Y. Yui, S. Miyazaki, A. Nakamoto, A. Namera and S. Inokuchi, Anal. Bioanal. Chem., 2011, 400, 25-31.

107 A. Namera, A. Nakamoto, M. Nishida, T. Saito, I. Kishiyama, S. Miyazaki, M. Yahata, M. Yashiki and M. Nagao, J. Chromatogr. A, 2008, 1208, 71-75.

108 T. Saito, S. Morita, I. Kishiyama, S. Miyazaki, A. Nakamoto, M. Nishida, A. Namera, M. Nagao and S. Inokuchi, J. Chromatogr. B: Anal. Technol. Biomed. Life Sci, 2008, 872, 186-190.

109 A. Namera, T. Saito, S. Miyazaki, S. Ohta, H. Oikawa, A. Torikoshi, H. Shiraishi and M. Nagao, Forensic Toxicol., 2013, 31, 312-321.

110 T. Saito, H. Aoki, A. Namera, H. Oikawa, S. Miyazaki, A. Nakamoto and S. Inokuchi, Analytical sciences: the international journal of the Japan Society for Analytical Chemistry, 2011, 27, 999-1005.

111 T. Saito, N. Miura, A. Namera, S. Miyazaki, S. Ohta, H. Oikawa and S. Inokuchi, Chromatographia, 2013, 76, 781-789.

112 A. Namera, S. Yamamoto, T. Saito, S. Miyazaki, H. Oikawa, A. Nakamoto and M. Nagao, J. Sep. Sci., 2011, 34, 2232-2239.

113 M. Abdel-Rehim, J. Chromatogr. A, 2010, 1217, 2569-2580.

114 S. Magiera, J. Chromatogr. B: Anal. Technol. Biomed. Life Sci, 2013, 938, 86-95.

115 A. Abdel-Rehim and M. Abdel-Rehim, Biomed. Chromatogr., 2013, 27, 1188-1191.

116 S. Peters, E. Kaal, I. Horsting and H. G. Janssen, J. Chromatogr. A, 2012, 1226, 71-76.

117 R. Said, A. Pohanka, M. Abdel-Rehim and O. Beck, J. Chromatogr. B: Anal. Technol. Biomed. Life Sci, 2012, 897, 42-49.

118 S. Rani, A. Kumar, A. K. Malik and B. Singh, Chromatographia, 2011, 74, 235-242.

119 B. M. da Fonseca, I. E. D. Moreno, M. Barroso, S. Costa, J. A. Queiroz and E. Gallardo, Anal. Bioanal. Chem., 2013, 405, 3953-3963.

120 R. Said, A. Pohanka, M. Andersson, O. Beck and M. AbdelRehim, J. Chromatogr. B: Anal. Technol. Biomed. Life Sci, 2011, 879, 815-818.

121 S. M. Daryanavard, A. Jeppsson-Dadoun, L. I. Andersson, M. Hashemi, A. Colmsjo and M. Abdel-Rehim, Biomed. Chromatogr., 2013, 27, 1481-1488.

122 X. J. Kang, C. Pan, Q. Xu, Y. F. Yao, Y. Wang, D. J. Qi and Z. Z. Gu, Anal. Chim. Acta, 2007, 587, 75-81.

123 F. Augusto, L. W. Hantao, N. G. S. Mogollon and S. C. G. N. Braga, TrAC, Trends Anal. Chem., 2013, 43, 14-23. 
124 C. Y. Shi, J. R. Meng and C. H. Deng, J. Mater. Chem., 2012, 22, 20778-20785.

125 X. L. Hou, Y. L. Wu, T. Yang and X. D. Du, J. Chromatogr. B: Anal. Technol. Biomed. Life Sci, 2013, 929, 107-115.

126 M. M. Li, X. G. Liu, F. S. Dong, J. Xu, Z. Q. Kong, Y. B. Li and Y. Q. Zheng, J. Chromatogr. A, 2013, 1300, 95-103.

127 X. D. Du, Y. L. Wu, H. J. Yang and T. Yang, J. Chromatogr. A, 2012, 1260, 25-32.

128 T. T. Ho, Z. G. Li, H. Y. Lin and M. R. Lee, J. Chin. Chem. Soc., 2013, 60, 1033-1042.

129 X. Li, S. Y. Xu, C. S. Pan, H. J. Zhou, X. G. Jiang, Y. Mang, M. L. Ye and H. F. Zou, J. Sep. Sci., 2007, 30, 930-943.

130 B. Suarez, B. M. Simonet, S. Cardenas and M. Valcarcel, J. Chromatogr. A, 2007, 1159, 203-207.

131 Z. H. Zhang, X. Yang, X. Chen, M. L. Zhang, L. J. Luo, M. J. Peng and S. Z. Yao, Anal. Bioanal. Chem., 2011, 401, 2855-2863.

132 O. Nunez, H. Gallart-Ayala, C. P. B. Martins, P. Lucci and R. Busquets, J. Chromatogr. B: Anal. Technol. Biomed. Life Sci, 2013, 927, 3-21.

133 S. H. Yang, H. Fan, R. J. Classon and K. A. Schug, J. Sep. Sci., 2013, 36, 2922-2938.

134 S. Souverain, S. Rudaz and J. -L. Veuthey, J. Chromatogr. B: Anal. Technol. Biomed. Life Sci., 2004, 801, 141-156.

135 X. L. Zhou, X. Y. Ye and A. M. Calafat, J. Chromatogr. B: Anal. Technol. Biomed. Life Sci, 2012, 881-82, 27-33.

136 V. Neu, N. Delmotte, U. Kobold, T. Dulffer, R. Herrmann, H. von der Eltz and C. G. Huber, Anal. Bioanal. Chem., 2012, 404, 863-874.

137 L. Ye, Q. Wang, J. P. Xu, Z. G. Shi and L. Xu, J. Chromatogr. A, 2012, 1244, 46-54.

138 Y. Sato, E. Yamamoto, S. Takakuwa, T. Kato and N. Asakawa, J. Chromatogr. A, 2008, 1190, 8-13.

139 E. Yamamoto, S. Takakuwa, T. Kato and N. Asakawa, J. Chromatogr. B: Anal. Technol. Biomed. Life Sci, 2007, 846, 132-138.

140 K. Bentayeb, R. Batlle, C. Sanchez, C. Nerin and C. Domeno, J. Chromatogr. B: Anal. Technol. Biomed. Life Sci, 2008, 869, 1-8.

141 F. Gasparrini, G. Cancelliere, A. Ciogli, I. D'Acquarica, D. Misiti and C. Villani, J. Chromatogr. A, 2008, 1191, 205213.

142 N. Delaunay-Bertoncini, V. Pichon and M. C. Hennion, Chromatographia, 2001, 53, S224-S230.

143 A. R. Chaves and M. E. Queiroz, J. Chromatogr. B: Anal. Technol. Biomed. Life Sci., 2013, 928, 37-43.

144 B. Winther, M. Nordlund, E. Paus, L. Reubsaet and T. G. Halvorsen, J. Sep. Sci., 2009, 32, 2937-2943.

145 N. W. Turner, S. Subrahmanyam and S. A. Piletsky, Anal. Chim. Acta, 2009, 632, 168-180.
146 C. Yuan, J. Kosewick, X. He, M. Kozak and S. H. Wang, Rapid Commun. Mass Spectrom., 2011, 25, 12411249.

147 Y. L. Hu, J. L. Pan, K. G. Zhang, H. X. Lian and G. K. Li, TrAC, Trends Anal. Chem., 2013, 43, 37-52.

148 E. V. Piletska, R. Burns, L. A. Terry and S. A. Piletsky, J. Agric. Food Chem., 2012, 60, 95-99.

149 S. Piletsky, E. Piletska, K. Karim, G. Foster, C. Legge and A. Turner, Anal. Chim. Acta, 2004, 504, 123-130.

150 A. Martin-Esteban, TrAC, Trends Anal. Chem., 2013, 45, 169181.

151 L. G. Chen and B. Li, Food Chem., 2013, 141, 23-28.

152 Z. M. Zhang, W. Tan, Y. L. Hu and G. K. Li, J. Chromatogr. A, 2011, 1218, 4275-4283.

153 Z. G. Xu, C. Y. Song, Y. L. Hu and G. K. Li, Talanta, 2011, 85, 97-103.

154 R. Jackson, I. Petrikovics, E. P. C. Lai and J. C. C. Yu, Anal. Methods, 2010, 2, 552-557.

155 E. C. Figueiredo, R. Sparrapan, G. B. Sanvido, M. G. Santos, M. A. Z. Arruda and M. N. Eberlin, Analyst, 2011, 136, 37533757.

156 S. V. Duy, I. Lefebvre-Tournier, V. Pichon, F. HugonChapuis, J. Y. Puy and C. Perigaud, J. Chromatogr. B: Anal. Technol. Biomed. Life Sci, 2009, 877, 1101-1108.

157 V. Thibert, P. Legeay, F. Chapuis-Hugon and V. Pichon, Talanta, 2012, 88, 412-419.

158 N. Harun, R. A. Anderson and P. A. G. Cormack, Anal. Bioanal. Chem., 2010, 396, 2449-2459.

159 B. T. S. Bui, F. Merlier and K. Haupt, Anal. Chem., 2010, 82, 4420-4427.

160 H. Hou, X. Zhang, Y. Tian, G. Tang, Y. Liu and Q. Hu, J. Pharm. Biomed. Sci., 2012, 63, 17-22.

161 M. Szultka, J. Szeliga, M. Jackowski and B. Buszewski, Anal. Bioanal. Chem., 2012, 403, 785-796.

162 M. McKeague, C. R. Bradley, A. De Girolamo, A. Visconti, J. D. Miller and M. C. Derosa, Int. J. Mol. Sci., 2010, 11, 4864-4881.

163 E. Luzi, M. Minunni, S. Tombelli and M. Mascini, TrAC, Trends Anal. Chem., 2003, 22, 810-818.

164 B. Madru, F. Chapuis-Hugon, E. Peyrin and V. Pichon, Anal. Chem., 2009, 81, 7081-7086.

165 B. Madru, F. Chapuis-Hugon and V. Pichon, Talanta, 2011, 85, 616-624.

166 S. N. Aslipashaki, T. Khayamian and Z. Hashemian, J. Chromatogr. B: Anal. Technol. Biomed. Life Sci., 2013, 925, 26-32.

167 J. H. Kim, E. T. Hwang, K. K. Kang, R. Tatavarty and M. B. Gu, J. Mater. Chem., 2011, 21, 19203-19206.

168 O. Veiseh, J. W. Gunn and M. Q. Zhang, Adv. Drug Delivery Rev., 2010, 62, 284-304. 